\title{
Remarks on Pressure Blow-Up Criterion of the 3D Zero-Diffusion Boussinesq Equations in Margin Besov Spaces
}

\author{
Min Fu ${ }^{1,2}$ and Chao Cai ${ }^{1}$ \\ ${ }^{1}$ State Key Laboratory for Multispectral Information Processing Technologies, School of Automation, \\ Huazhong University of Science and Technology, Wuhan, China \\ ${ }^{2}$ College of Science, Wuhan Institute of Technology, Wuhan, China \\ Correspondence should be addressed to Chao Cai; caichao@hust.edu.cn
}

Received 13 February 2017; Accepted 6 March 2017; Published 24 April 2017

Academic Editor: Pavel Kurasov

Copyright (C) 2017 Min Fu and Chao Cai. This is an open access article distributed under the Creative Commons Attribution License, which permits unrestricted use, distribution, and reproduction in any medium, provided the original work is properly cited.

\begin{abstract}
This study is focused on the pressure blow-up criterion for a smooth solution of three-dimensional zero-diffusion Boussinesq equations. With the aid of Littlewood-Paley decomposition together with the energy methods, it is proved that if the pressure satisfies the following condition on margin Besov spaces, $\pi(x, t) \in L^{2 /(2+r)}\left(0, T ; \dot{B}_{\infty, \infty}^{r}\right)$ for $r= \pm 1$, then the smooth solution can be continually extended to the interval $\left(0, T^{*}\right)$ for some $T^{*}>T$. The findings extend largely the previous results.
\end{abstract}

\section{Introduction and Main Results}

It is well known that mathematical models in fluid dynamics have attracted more and more attention in the past ten years [1]. In this paper, we consider the dynamical models of the ocean or the atmosphere which arise from the density dependent incompressible Navier-Stokes equations by using the so-called Boussinesq approximation [2]. The so-called Boussinesq system is governed by the following nonlinear partial differential equations:

$$
\begin{aligned}
\partial_{t} u-\mu \Delta u+(u \cdot \nabla) u+\nabla \pi & =\theta e_{3}, \\
\partial_{t} \theta-\kappa \Delta \theta+(u \cdot \nabla) \theta & =0, \\
\nabla \cdot u & =0,
\end{aligned}
$$

associated with the initial conditions

$$
\begin{aligned}
& u(x, 0)=u_{0}, \\
& \theta(x, 0)=\theta_{0} .
\end{aligned}
$$

Here, $u(x, t), \theta(x, t)$, and $\pi(x, t)$ represent the unknown velocity vector field, temperature field, and the unknown pressure scalar field, respectively. The constant $\mu>0$ is the kinematic viscosity and the constant $\kappa \geq 0$ is the thermal diffusivity. The quantities $u_{0}(x)$ and $\theta_{0}(x)$ are the given initial velocity and initial temperature, respectively.

As an important mathematical model in many geophysical applications, the Boussinesq system attracted more and more attention in the past ten years [3-6]. Moreover, when the temperature field $\theta=0$, it reduces to the classic NavierStokes equations (see [7])

$$
\begin{aligned}
\partial_{t} u-\mu \Delta u+(u \cdot \nabla) u+\nabla \pi & =0, \\
\nabla \cdot u & =0 .
\end{aligned}
$$

In the three-dimensional case, the same as the classic Navier-Stokes equations, the issue on the global smooth solution with large initial data is still a challenging open problem. The development of blow-up criteria is of importance for both theoretical and practical situations [8-10]. When $\nu, \kappa>0$, the first blow-up criterion of 3D Boussinesq equations in Lebesgue space was considered by Ishimura and Morimoto [11]; they proved that if the velocity satisfies

$$
\nabla u \in L^{1}\left(0, T ; L^{\infty}\left(\mathbf{R}^{3}\right)\right)
$$

then the smooth solution can be continually extended to the interval $\left(0, T^{*}\right)$ for some $T^{*}>T$. For the zero-viscosity case, 
that is, $\mu=0, \kappa>0$, Fan and Zhou [12] studied the blow-up criterion:

$$
\nabla \times u \in L^{1}\left(0, T ; \dot{B}_{\infty, \infty}^{0}\left(\mathbf{R}^{3}\right)\right) .
$$

In the zero-diffusion case, that is, $\mu>0, \kappa=0$, the situation becomes more difficult. The main obstacle is that the temperature function $\theta(x, t)$ in the transport equation does not gain any smoothness. Hence, the blow-up issue of the zero-diffusive Boussinesq equations (1) with $\kappa=0$ is more difficult than that of full viscous Boussinesq system (1). Jia et al. [13] recently studied the blow-up criterion for local smooth solutions of zero-diffusive Boussinesq equations (1) in the large critical Besov space

$$
\int_{0}^{T}\|u\|_{B_{q, \infty}^{s}\left(\mathbf{R}^{3}\right)}^{p} d s<\infty
$$

with

$$
\begin{aligned}
& \frac{2}{p}+\frac{3}{q}=1+s, \\
& \frac{3}{1+s}<p \leq \infty, \quad-1<s \leq 1,(p, s) \neq(\infty, 1) .
\end{aligned}
$$

Recently, Wang [14] also proved the blow-up criterion for the zero-diffusive Boussinesq equation when the velocity components satisfy

$$
\begin{aligned}
\int_{0}^{T} \frac{\left\|u_{1}|+| u_{2} \mid\right\|_{L^{p, \infty}}^{q}}{1+\ln \left(e+\|\nabla u\|_{L^{2}}^{2}\right)} d s<\infty, & \\
& \frac{2}{q}+\frac{3}{p}=1,3<p<\infty .
\end{aligned}
$$

For the pressure blow-up criterion of the zero-diffusive Boussinesq equations (1), Dong et al. [15] investigated the pressure regular criterion when

$$
\pi(x, t) \in L^{2 /(2+r)}\left(0, T ; \dot{B}_{\infty, \infty}^{r}\left(\mathbf{R}^{3}\right)\right) \quad \text { for }-1<r<1 .
$$

However, the methods in [15] are not available for the margin case $r=1$ or $r=-1$. One may also refer to some important regularity criteria on the fluid dynamics $[16,17]$.

The aim of the present paper is to improve the pressure blow-up criterion for smooth solution of three-dimensional zero-diffusion Boussinesq equations in the margin Besov spaces $r= \pm 1$ in (9); more precisely, we will prove the following result.

Theorem 1. Suppose $\mu>0, \kappa=0$. Let $T>0 ;(u, \theta)$ is a smooth solution of zero-diffusion Boussinesq equations (1) with $\left(u_{0}, \theta_{0}\right) \in H^{m}\left(\mathbf{R}^{3}\right), m>3 / 2$. If the pressure $\pi$ satisfies

$$
\pi(x, t) \in L^{2 /(2+r)}\left(0, T ; \dot{B}_{\infty, \infty}^{r}\left(\mathbf{R}^{3}\right)\right) \quad \text { for } r= \pm 1,
$$

then the smooth solution can be continually extended to the interval $\left(0, T^{*}\right)$ for some $T^{*}>T$.

Theorem 1 also implies the following corollary.
Corollary 2. Suppose $(u, \theta)$ is the smooth solution of zerodiffusion Boussinesq equations satisfying

$$
\begin{aligned}
& u \in C\left(\left[0, T_{1}\right) ; H^{m}\left(\mathbf{R}^{3}\right)\right) \cap L^{2}\left(0, T_{1} ; H^{m+1}\left(\mathbf{R}^{3}\right)\right), \\
& \theta \in C\left(\left[0, T_{1}\right) ; H^{m}\left(\mathbf{R}^{3}\right)\right) .
\end{aligned}
$$

If $T$ is the maximal existence time of the smooth solution, then

$$
\begin{array}{r}
T<\infty \Longrightarrow \\
\int_{0}^{T}\|\pi\|_{\dot{B}_{\infty, \infty}^{r}}^{2 /(2+r)} d s=+\infty,
\end{array}
$$

where $r= \pm 1$.

Remark 3. It should be mentioned that since our work spaces here are margin cases in Besov spaces, the methods used by Dong et al. [15] where the finding is mainly based on the function spaces decomposition cannot be available any more. Furthermore, compared with the many previous results on the pressure regularity criterion for full viscous fluid dynamical models such as Navier-Stokes equations and MHD equations (see [18]), the zero-diffusion Boussinesq equations (1) do not have the important inequality

$$
\|\pi\|_{L^{p}} \leq C\|u\|_{L^{2 p}}^{2}, \quad 1<p<\infty,
$$

due to the appearance of $\theta e_{3}$. In order to overcome the difficulty of the absence of the above estimate, we will give some more explicit estimates on the gradient of the pressure in this paper. Additionally, our results here more or less extended the previous results by Gala et al. $[19,20]$ and others [14]. Since it is also interesting and important to consider this issue in some working space such as Morrey Space (see $[17,21,22])$, we will focus on this problem in the forthcoming paper.

\section{Preliminary}

In this section, $C$ stands for a generic positive constant which may vary from line to line. $L^{p}\left(\mathbf{R}^{3}\right)$ with $1 \leq p \leq \infty$ denotes the usual Lebesgue space and $H^{s}\left(\mathbf{R}^{3}\right)$ with $s \in \mathbf{R}$ is the inhomogeneous fractional Sobolev space with the norm

$$
\|f\|_{H^{s}}=\left(\int_{\mathbf{R}^{3}}\left|(I-\Delta)^{s / 2} f\right|^{2} d \xi\right)^{1 / 2} .
$$

We now provide the Littlewood-Paley decomposition (see [23]). Let $\mathcal{S}\left(\mathbf{R}^{3}\right)$ be the Schwartz class of a rapidly decreasing function. Given $f \in \mathcal{S}\left(\mathbf{R}^{3}\right)$, we define the Fourier transformation $\mathscr{F} f=\widehat{f}$ as

$$
\widehat{f}(\xi)=\int_{\mathbf{R}^{3}} e^{-i x \cdot \xi} f(x) d x .
$$

Take two nonnegative radial functions $\chi, \psi \in \mathcal{S}\left(\mathbf{R}^{3}\right)$ supported, respectively, in $\mathscr{B}=\left\{\xi \in \mathbf{R}^{3}:|\xi| \leq 4 / 3\right\}$ and $\mathscr{C}=\left\{\xi \in \mathbf{R}^{3}: 3 / 4 \leq|\xi| \leq 8 / 3\right\}$, such that

$$
\sum_{j \in Z} \psi\left(2^{-j} \xi\right)=1, \quad \xi \in \mathbf{R}^{3} \backslash\{0\} .
$$


Let $h=\mathscr{F}^{-1} \psi$ and $\widetilde{h}=\mathscr{F}^{-1} \chi$. The frequency localization operator is defined by

$$
\begin{aligned}
& \Delta_{j} f=\psi\left(2^{-j} D\right) f=2^{3 j} \int_{\mathbf{R}^{3}} h\left(2^{j} y\right) f(x-y) d y, \\
& S_{j} f=\chi\left(2^{-j} D\right) f=\sum_{-1 \leq k \leq j-1} \Delta_{k} f, \\
&=2^{3 j} \int_{\mathbf{R}^{3}} \widetilde{h}\left(2^{j} y\right) f(x-y) d y, \\
& \Delta_{1} f=S_{0} f, \Delta_{j} f=0 \text { for } j \leq-2 .
\end{aligned}
$$

Formally, $\Delta_{j}$ is a frequency projection to the annulus $\left\{|\xi| \approx 2^{j}\right\}$, and $S_{j}$ is a frequency projection to the ball $\left\{|\xi| \lesssim 2^{j}\right\}$. The aforementioned dyadic decomposition has nice quasi-orthogonality, with the choice of $\chi$ and $\psi$; namely, given any $\chi, \psi \in \mathcal{S}\left(\mathbf{R}^{3}\right)$, we have the following properties:

$$
\begin{aligned}
\Delta_{i} \Delta_{j} f & =0, \quad \text { if }|i-j| \geq 2, \\
\Delta_{i}\left(S_{j-1} \Delta_{j} g\right) & =0, \quad \text { if }|i-j| \geq 5 .
\end{aligned}
$$

With the full-dyadic decomposition, we define the homogeneous Besov space $\dot{B}_{p, q}^{s}\left(\mathbf{R}^{3}\right)$ :

$$
\dot{B}_{p, q}^{s}\left(\mathbf{R}^{3}\right)=\left\{f \in \frac{\mathcal{S}^{\prime}\left(\mathbf{R}^{3}\right)}{\mathscr{P}\left(\mathbf{R}^{3}\right)}:\|f\|_{\dot{B}_{p, q}^{s}}<\infty\right\},
$$

where

$$
\|f\|_{\dot{B}_{p, q}^{s}}= \begin{cases}\left(\sum_{j=-\infty}^{\infty} 2^{j s q}\left\|\Delta_{j} f\right\|_{L_{p}}^{q}\right)^{1 / q}, & 1 \leq q<\infty, \\ \sup _{j \in Z} 2^{j s}\left\|\Delta_{j} f\right\|_{L_{p}}, & q=\infty .\end{cases}
$$

The set $\mathcal{S}^{\prime}\left(\mathbf{R}^{3}\right)$ of tempered distributions is the dual set and $\mathscr{P}\left(\mathbf{R}^{3}\right)$ is the polynomials space.

In Lemma 4, we recall the Bernstein inequalities which will be applied frequently.

Lemma 4 (Chemin [24]). Suppose $k, j \in Z$ and $1 \leq p \leq q \leq$ $\infty$; one has, for all $f \in \mathcal{S}\left(\mathbf{R}^{3}\right)$,

$$
\sup _{|\alpha|=k}\left\|\nabla^{\alpha} \Delta_{j} f\right\|_{L_{q}} \leq C 2^{j k+3 j(1 / p-1 / q)}\left\|\Delta_{j} f\right\|_{L_{p}},
$$

where $C$ are the positive constants independent of $j, k$.

Lemma 5 (Meyer [25]). For any function $f$ belonging to the homogeneous Sobolev space $\dot{H}^{1}\left(\mathbf{R}^{3}\right)$, one has

$$
\|f\|_{L^{4}}^{2} \leq C\|f\|_{B}\|\nabla f\|_{L^{2}},
$$

where $B$ is the homogeneous Besov space $\dot{B}_{\infty, \infty}^{-1}\left(\mathbf{R}^{3}\right)$.
Lemma 6 (Kato-Ponce [26]). Suppose $f, g \in W^{k, p}\left(\mathbf{R}^{3}\right), 1<$ $p<\infty$, for $1 \leq s \leq k$; then,

$$
\begin{aligned}
\left\|J^{s}(f g)-f J^{s} g\right\|_{L^{p}} \leq & C\|\nabla f\|_{L^{q}}\left\|J^{s-1} g\right\|_{L^{r}} \\
& +C\|g\|_{L^{q}}\left\|J^{s} f\right\|_{L^{r}},
\end{aligned}
$$

where $J^{s}=(I-\Delta)^{s / 2}, 1 / p=1 / q+1 / r$, and $1<q<\infty, 1<$ $r<\infty$.

\section{Proof of Theorem 1}

Step 1 (energy estimate). Multiplying both sides of the second equation of zero-diffusion Boussinesq equations (1) by $|\theta|^{p-2} \theta$ and integrating in $\mathbf{R}^{3}$ give

$$
\frac{1}{p} \frac{d}{d t}\|\theta(t)\|_{L^{p}}^{p}=0
$$

Integrating in time,

$$
\|\theta(t)\|_{L^{p}} \leq\left\|\theta_{0}\right\|_{L^{p}}
$$

where we have used $\int_{\mathbf{R}^{3}}(u \cdot \nabla) \theta \theta d x=0$.

Taking the inner product to the first equation of (1) by $u$ and applying the integration by parts yield

$$
\begin{aligned}
& \frac{1}{2} \frac{d}{d t}\|u(t)\|_{L^{2}}^{2}+\mu\|\nabla u\|_{L^{2}}^{2} \leq\|\theta\|_{L^{2}}\|u\|_{L^{2}} \\
& \quad \leq\left\|\theta_{0}\right\|_{L^{2}}\|u\|_{L^{2}} ;
\end{aligned}
$$

therefore, it follows from Gronwall inequality that

$$
\sup _{0<t<T}\|u(t)\|_{L^{2}}^{2}+\mu \int_{0}^{T}\|\nabla u\|_{L^{2}}^{2} d t \leq C .
$$

Since the pressure here plays an important role in our argument, we apply the operator $\nabla$ div to the first equation of (1) to get

$\nabla \pi$

$$
=(-\Delta)^{-1} \sum_{i, j=1}^{3} \frac{\partial^{2}}{\partial x_{i} \partial x_{j}}\left(\nabla\left(u_{i} u_{j}\right)-(-\Delta)^{-1}\left(\nabla \partial_{3} \theta_{3}\right)\right),
$$

and then, according to Calderon-Zygmund inequality and (27), we obtain

$$
\|\nabla \pi\|_{L^{p}} \leq C\|\| u\left|\nabla u\left\|_{L^{p}}+C\right\| \theta\left\|_{L^{p}} \leq C\right\|\|u \mid \nabla u\|_{L^{p}}+C,\right.
$$


Step 2 ( $L^{4}$ estimate of $u$ ). Taking the inner product to the first equation, the second equation of (1) by $|u|^{2} u$ and $|\theta|^{2} \theta$, respectively, employing Hölder inequality and Young inequality, we have

$$
\begin{aligned}
& \frac{1}{4} \frac{d}{d t}\left(\|u\|_{L^{4}}^{4}+\|\theta\|_{L^{4}}^{4}\right)+\mu\|u \mid \nabla u\|_{L^{2}}^{2} \\
& \quad \leq \int_{\mathbf{R}^{3}} \theta e_{3} \cdot u|u|^{2} d x-\int_{\mathbf{R}^{3}} u \cdot \nabla \pi|u|^{2} d x \\
& \quad \leq C\|\theta\|_{L^{4}}\|u\|_{L^{4}}^{3}-\int_{\mathbf{R}^{3}} u \cdot \nabla \pi|u|^{2} d x \\
& \quad \leq C\left(\|u\|_{L^{4}}^{4}+\|\theta\|_{L^{4}}^{4}\right)-\int_{\mathbf{R}^{3}} u \cdot \nabla \pi|u|^{2} d x \\
& \quad=C\left(\|u\|_{L^{4}}^{4}+\|\theta\|_{L^{4}}^{4}\right)+I,
\end{aligned}
$$

where we have used

$$
\begin{aligned}
& \int_{\mathbf{R}^{3}}(u \cdot \nabla u) \cdot|u|^{2} u d x=0, \\
& \int_{\mathbf{R}^{3}}(u \cdot \nabla \theta) \cdot|\theta|^{2} \theta d x=0 .
\end{aligned}
$$

Case $1(r=1)$. That is,

$$
\begin{aligned}
& \int_{0}^{T}\|\pi(t)\|_{B_{\infty, \infty}^{1}}^{2 / 3} d t<\infty \\
& I \leq\left.\left|\int_{\mathbf{R}^{3}} u \cdot \nabla \pi\right| u\right|^{2} d x \mid \\
& =\left.\left|\int_{\mathbf{R}^{3}}\left(\sum_{j<-N}+\sum_{j=-N}^{N}+\sum_{j>N}\right) \Delta_{j}(\nabla \pi) \cdot\right| u\right|^{2} u d x \mid \\
& \leq\left.\left|\int_{\mathbf{R}^{3}} \sum_{j<-N} \Delta_{j}(\nabla \pi) \cdot\right| u\right|^{2} u d x \mid \\
& \quad+\left.\left|\int_{\mathbf{R}^{3}} \sum_{j=-N}^{N} \Delta_{j}(\nabla \pi) \cdot\right| u\right|^{2} u d x \mid \\
& \quad+\left.\left|\int_{\mathbf{R}^{3}} \sum_{j>N} \Delta_{j}(\nabla \pi) \cdot\right| u\right|^{2} u d x \mid=I_{1}+I_{2}+I_{3}
\end{aligned}
$$

for an arbitrary positive integer $N$.
In order to estimate the term $I_{1}$, we apply Hölder inequality, Lemma 5, and Young inequality:

$$
\begin{aligned}
I_{1} & \leq \sum_{j<-N}\left\|\Delta_{j} \nabla \pi\right\|_{L^{4}}\|u\|_{L^{4}}^{3} \\
& \leq \sum_{j<-N} 2^{3 j(1 / 2-1 / 4)}\left\|\Delta_{j} \nabla \pi\right\|_{L^{2}}\|u\|_{L^{4}}^{3} \\
& \leq\left(\sum_{j<-N} 2^{(3 / 2) j}\right)^{1 / 2} \sum_{j<-N}\left(\left\|\Delta_{j} \nabla \pi\right\|_{L^{2}}^{2}\right)^{1 / 2}\|u\|_{L^{4}}^{3} \\
& \leq 2^{-3 N / 4}\|\nabla \pi\|_{L^{2}}\|u\|_{L^{4}}^{3} \\
& \leq 2^{-3 N / 4}\left(\|u \mid \nabla u\|_{L^{2}}+C\right)\|u\|_{L^{4}}^{3} \\
& \leq C\left(2^{-N / 4}\|u\|_{L^{4}}\right)^{3}\||u| \nabla u\|_{L^{2}}+C\left(2^{-N / 4}\|u\|_{L^{4}}\right)^{3} .
\end{aligned}
$$

In order to estimate the term $I_{2}$, we apply Hölder inequality and Young inequality:

$$
\begin{aligned}
I_{2} & \leq \sum_{j=-N}^{N}\left\|\Delta \Delta_{j} \nabla \pi\right\|_{L^{4}}\|u\|_{L^{4}}^{3} \\
& \leq N^{3 / 4}\|\nabla \pi\|_{B_{\infty, \infty}^{0}}^{1 / 2}\|\nabla \pi\|_{L^{2}}^{1 / 2}\|u\|_{L^{4}}^{3} \\
& \leq C\left(N\|\nabla \pi\|_{B_{\infty}^{0}, \infty}^{2 / 3}\right)^{3 / 4}\left(\||u| \nabla u\|_{L^{2}}^{1 / 2}+C\right)\|u\|_{L^{4}}^{3} \\
& \leq C\left(N\|\nabla \pi\|_{B_{\infty, \infty}^{0}}^{2 / 3}\right)\|u\|_{L^{4}}^{4}+\frac{\mu}{8}\||u| \nabla u\|_{L^{2}}^{2}+C .
\end{aligned}
$$

For $I_{3}$, we apply Hölder inequality, Lemma 5, and (29):

$$
\begin{aligned}
I_{3} & \leq \sum_{j>N}\left\|\Delta_{j} \nabla \pi\right\|_{L^{4}}\|u\|_{L^{4}}^{3} \leq 2^{-3 N / 4}\|\nabla \pi\|_{L^{2}}\|u\|_{L^{4}}^{3} \\
& \leq C\left(2^{-N / 4}\|u\|_{L^{4}}\right)^{3}\left(\||u| \nabla u\|_{L^{2}}+C\right) \\
& \leq C\left(2^{-N / 4}\|u\|_{L^{4}}\right)^{3}\||u| \nabla u\|_{L^{2}}+C\left(2^{-N / 4}\|u\|_{L^{4}}\right)^{3} .
\end{aligned}
$$

We only need to choose the integer $N$ satisfying

$$
C\left(2^{-N / 4}\|u\|_{L^{4}}\right)^{3} \leq \frac{\mu}{8}
$$

For example, we may set

$$
N=\left[\frac{(1 / 3) \ln (\mu / 8 C)+\ln \left(\|u\|_{L^{4}}+e\right)}{\ln 2}\right]+1 .
$$

Combining $I_{1}, I_{2}$, and $I_{3}$ and the above inequality yields

$$
\begin{aligned}
& \frac{d}{d t}\left(\|u\|_{L^{4}}^{4}+\|\theta\|_{L^{4}}^{4}\right)+\mu\||u| \nabla u\|_{L^{2}}^{2} \\
& \quad \leq C\left(\|u\|_{L^{4}}^{4}+\|\theta\|_{L^{4}}^{4}\right)+C N\|\nabla \pi\|_{B_{\infty, \infty}^{0}}^{2 / 3}\|u\|_{L^{4}}^{4}+C,
\end{aligned}
$$


and the above inequality reduces to

$$
\begin{aligned}
& \frac{d}{d t}\left(\|u\|_{L^{4}}^{4}+\|\theta\|_{L^{4}}^{4}\right) \leq C\left(\|u\|_{L^{4}}^{4}+\|\theta\|_{L^{4}}^{4}\right) \\
& \quad+C N\|\nabla \pi\|_{B_{\infty, \infty}^{0}}^{2 / 3}\|u\|_{L^{4}}^{4}+C=\{C \\
& \left.\quad+C\left(\frac{\ln C+\ln \left(\|u\|_{L^{4}}^{4}+e\right)}{\ln 2}+1\right)\|\nabla \pi\|_{B_{\infty, \infty}^{0}}^{2 / 3}\right\} \\
& \quad \cdot\left(\|u\|_{L^{4}}^{4}+\|\theta\|_{L^{4}}^{4}\right)+C .
\end{aligned}
$$

Taking Gronwall inequality into consideration,

$$
\begin{aligned}
& \|u\|_{L^{4}}^{4}+\|\theta\|_{L^{4}}^{4} \leq\left(\left\|u_{0}\right\|_{L^{4}}^{4}+\left\|\theta_{0}\right\|_{L^{4}}^{4}+C T\right) \exp \{C T \\
& +\int_{0}^{T} C\left(\frac{\ln C+\ln \left(\|u\|_{L^{4}}^{4}+e\right)}{\ln 2}+1\right) \\
& \left.\cdot\|\nabla \pi\|_{B_{\infty, \infty}^{0}}^{2 / 3} d \tau\right\} \leq\left(\left\|u_{0}\right\|_{L^{4}}^{4}+\left\|\theta_{0}\right\|_{L^{4}}^{4}+C T\right) \\
& \cdot \exp \left\{C T+\int_{0}^{T} C\left[\ln \left(\|u\|_{L^{4}}^{4}+e\right)+C\right]\right. \\
& \left.\cdot\|\nabla \pi\|_{B_{\infty}^{0}, \infty}^{2 / 3} d \tau\right\}
\end{aligned}
$$

for a suitable constant $C$.

Thus, we also have

$$
\begin{aligned}
& \ln \left(\|u\|_{L^{4}}^{4}+\|\theta\|_{L^{4}}^{4}+e\right) \\
& \leq \ln \left(\left\|u_{0}\right\|_{L^{4}}^{4}+\left\|\theta_{0}\right\|_{L^{4}}^{4}+C T\right)+C T \\
& \quad+\int_{0}^{T} C\left[\ln \left(\|u\|_{L^{4}}^{4}+e\right)+C\right]\|\nabla \pi\|_{B_{\infty, \infty}^{0}}^{2 / 3} d \tau .
\end{aligned}
$$

Employing Gronwall inequality again, one shows that

$$
\begin{aligned}
& \ln \left(\|u\|_{L^{4}}^{4}+\|\theta\|_{L^{4}}^{4}+e\right) \\
& \quad \leq\left(\ln \left(\left\|u_{0}\right\|_{L^{4}}^{4}+\left\|\theta_{o}\right\|_{L^{4}}^{4}+C T\right)+C\right) \\
& \quad \cdot \exp \left(\int_{0}^{T} C\|\nabla \pi\|_{B_{\infty, \infty}^{0}}^{2 / 3} d \tau\right)<\infty .
\end{aligned}
$$

Case $2(r=-1)$. That is,

$$
\int_{0}^{T}\|\pi(t)\|_{\dot{B}_{\infty, \infty}^{-1}}^{2} d t<\infty
$$

Employing Hölder inequality, Young inequality, and Lemma 5, one shows that

$$
\begin{aligned}
I & =2 \int_{\mathrm{R}^{3}} \pi u|u| \cdot \nabla u d x \\
& \leq C\|\pi\|_{L^{4}}^{2}\|u\|_{L^{4}}^{2}+\frac{\mu}{8}\||u| \nabla u\|_{L^{2}}^{2} \\
& \leq C\|\pi\|_{B_{\infty, \infty}^{-1}}\|\nabla p\|_{L^{2}}\|u\|_{L^{4}}^{2}+\frac{\mu}{8}\||u| \nabla u\|_{L^{2}}^{2} \\
& \leq C\|\pi\|_{B_{\infty}^{-1}, \infty}\left(C+\||u| \nabla u\|_{L^{2}}\right)\|u\|_{L^{4}}^{2}+\frac{\mu}{8}\||u| \nabla u\|_{L^{2}}^{2} \\
& \leq C\|\pi\|_{B_{\infty}^{-1}, \infty}^{2}\|u\|_{L^{4}}^{4}+\frac{\mu}{4}\||u| \nabla u\|_{L^{2}}^{2}+C .
\end{aligned}
$$

Inserting it into (30), we get

$$
\begin{aligned}
& \frac{d}{d t}\left(\|u\|_{L^{4}}^{4}+\|\theta\|_{L^{4}}^{4}\right)+\mu\||u| \nabla u\|_{L^{2}}^{2} \\
& \quad \leq C\left(\|\theta\|_{L^{4}}^{4}+\|u\|_{L^{4}}^{4}\right)+C\|\pi\|_{B_{\infty, \infty}^{-1}}^{2}\|u\|_{L^{4}}^{4} \\
& \quad \leq\left(C+C\|\pi\|_{B_{\infty, \infty}^{-1}}^{2}\right)\left(\|u\|_{L^{4}}^{4}+\|\theta\|_{L^{4}}^{4}\right) .
\end{aligned}
$$

Taking Gronwall inequality into consideration, one shows that

$$
\begin{aligned}
& \sup _{0<t<T}\left(\|u(t)\|_{L^{4}}^{4}+\|\theta\|_{L^{4}}^{4}\right)+\mu \int_{0}^{T}\||u| \nabla u\|_{L^{2}}^{2} d t \\
& \quad \leq\left(\left\|u_{0}\right\|_{L^{4}}^{4}+\left\|\theta_{0}\right\|_{L^{4}}^{4}\right) \exp \left\{\int_{0}^{T} C+C\|\pi(t)\|_{\dot{B}_{\infty, \infty}^{-1}}^{2} d t\right\} \\
& \quad \leq C\left(\left\|u_{0}\right\|_{L^{4}}^{4}+\left\|\theta_{0}\right\|_{L^{4}}^{4}\right) \exp \left\{\int_{0}^{T}\|\pi(t)\|_{\dot{B}_{\infty, \infty}^{-1}}^{2} d t\right\} \\
& \quad<\infty .
\end{aligned}
$$

From the estimates of $I$ in both Cases 1 and 2, we now derive the $L^{4}$ bound of the velocity $u$ :

$$
\|u\|_{L^{\infty}\left(0, T_{1} ; L^{4}\right)} \leq C .
$$

Step $3\left(H^{1}\right.$ estimates of $\left.u\right)$. Taking the inner product to the first equation of (1) by $\Delta u$ and applying the integral by parts yield

$$
\begin{aligned}
& \frac{1}{2} \frac{d}{d t}\|\nabla u\|_{L^{2}}^{2}+\mu\|\Delta u\|_{L^{2}}^{2} \\
& \quad=\int_{\mathbf{R}^{3}}(u \cdot \nabla) u \cdot \Delta u d x-\int_{\mathbf{R}^{3}} \theta e_{3} \cdot \Delta u d x \\
& \quad=: J_{1}+J_{2} .
\end{aligned}
$$


For $J_{1}, J_{2}$, we employ Hölder inequality, Young inequality, and Gagliardo-Nirenberg inequality:

$$
\begin{aligned}
J_{1} & =\int_{\mathbf{R}^{3}}(u \cdot \nabla) u \cdot \Delta u d x \leq C\|u\|_{L^{4}}\|\nabla u\|_{L^{4}}\|\Delta u\|_{L^{2}} \\
& \leq C\|u\|_{L^{4}}\|\nabla u\|_{L^{2}}^{1 / 4}\|\Delta u\|_{L^{2}}^{7 / 4} \\
& \leq \frac{\mu}{4}\|\Delta u\|_{L^{2}}^{2}+C\|\nabla u\|_{L^{2}}^{2} \\
J_{2} & \leq C\|\theta\|_{L^{2}}\|\Delta u\|_{L^{2}} \leq \frac{\mu}{4}\|\Delta u\|_{L^{2}}^{2}+C\|\theta\|_{L^{2}}^{2} \\
& \leq \frac{\mu}{4}\|\Delta u\|_{L^{2}}^{2}+C .
\end{aligned}
$$

Plugging (49) and (50) into (48) to deduce

$$
\frac{d}{d t}\|\nabla u\|_{L^{2}}^{2}+\mu\|\Delta u\|_{L^{2}}^{2} \leq C\|\nabla u\|_{L^{2}}^{2}+C
$$

taking Gronwall inequality into consideration together with (27), it follows that

$$
\sup _{0<t<T}\|\nabla u(t)\|_{L^{2}}^{2}+\int_{0}^{T}\|\Delta u\|_{L^{2}}^{2} \leq C ;
$$

that is

$$
\sup _{0<t<T}\|u(t)\|_{H^{1}}^{2}+\int_{0}^{T}\|\nabla u\|_{H^{1}}^{2} \leq C .
$$

Step $4\left(H^{m}\right.$ estimates of $\left.u, \theta\right)$. To prove the $H^{m}$ estimates of $u$, $\theta$, we need $L^{\infty}$ estimates of $\nabla u, \nabla \theta$. To do so, we first rewrite the first equations of (1):

$$
\partial_{t} u-\mu \Delta u=\theta e_{3}-u \cdot \nabla u-\nabla \pi
$$

Thanks to

$$
\begin{aligned}
& \int_{0}^{T}\left\|\theta e_{3}\right\|_{L^{3}}^{2} d s \leq\left\|\theta_{0}\right\|_{L^{3}}^{2} T, \\
& \int_{0}^{T}\|u \cdot \nabla u\|_{L^{3}}^{2} d s \leq \int_{0}^{T}\|u\|_{L^{6}}^{2}\|\nabla u\|_{L^{2}}^{2} d s \\
& \leq \operatorname{ess} \sup _{0<s<T}\|\nabla u(s)\|_{L^{2}}^{2} \int_{0}^{T}\|\Delta u\|_{L^{2}}^{2} d s \\
& \leq C \\
& \int_{0}^{T}\|\nabla p\|_{L^{3}}^{2} d s \leq \int_{0}^{T}\|u \cdot \nabla u\|_{L^{3}}^{2} d s+C T \\
& \leq \operatorname{ess} \sup _{0<s<T}\|\nabla u(s)\|_{L^{2}}^{2} \int_{0}^{T}\|\Delta u\|_{L^{2}}^{2} d s \\
& +C T \leq C T \text {, }
\end{aligned}
$$

the maximal regularity properties of the heat equation allow us to derive

$$
\int_{0}^{T}\|u\|_{W^{2,3}}^{2} d s \leq C
$$

implied by Sobolev imbedding inequality

$$
\int_{0}^{T}\|\nabla u\|_{L^{\infty}} d s \leq C .
$$

Now, we take the operator $\nabla$ into the second equation of (1) and then take the inner product by $|\nabla \theta|^{p-2} \nabla \theta$ to give

$$
\frac{d}{d t}\|\nabla \theta\|_{L^{p}} \leq C\|\nabla u\|_{L^{\infty}}\|\nabla \theta\|_{L^{p}} .
$$

Employing Gronwall inequality yields

$$
\|\nabla \theta\|_{L^{p}} \leq\left\|\nabla \theta_{0}\right\|_{L^{p}} \exp \left\{\int_{0}^{T}\|\nabla u\|_{L^{\infty}} d \tau\right\} .
$$

For any given $\epsilon, \rho>0$, we define the set $A_{\epsilon, \rho}(t)$ :

$$
A_{\epsilon, \rho}(t)=\left\{x \in \mathbf{R}^{3} ;|\nabla \theta|>\|\nabla \theta\|_{L^{\infty}}-\varepsilon,|x|>\rho\right\},
$$

and then

$$
\begin{aligned}
& \left(\|\nabla \theta(t)\|_{L^{\infty}}-\epsilon\right)\left|A_{\epsilon, \rho}(t)\right| \leq\|\nabla \theta\|_{L^{p}} \\
& \quad \leq\left\|\nabla \theta_{0}\right\|_{L^{2}}^{2 / p}\left\|\nabla \theta_{0}\right\|_{L^{\infty}}^{1-2 / p} \exp \left(\int_{0}^{T}\|\nabla u\|_{L^{\infty}} d \tau\right) \leq C,
\end{aligned}
$$

where $\left|A_{\epsilon, \rho}(t)\right|$ is the Lebesgue measure of the set $A_{\epsilon, \rho}(t)$. Since $C$ is independent of $t, \epsilon$, the above uniform bound allows us to take the limitation $t \rightarrow \infty, \epsilon \rightarrow 0$; then, we have

$$
\|\nabla \theta(t)\|_{L^{\infty}} \leq\left\|\nabla \theta_{0}\right\|_{L^{\infty}} \exp \left(\int_{0}^{T}\|\nabla u\|_{L^{\infty}} d \tau\right) .
$$

Taking the derivative operator $J^{m}=(I-\Delta)^{m / 2}$ into the first equation of (1) and taking the inner product by $J^{m} u$ and then applying Lemma 6, Hölder inequality, and Young inequality,

$$
\begin{aligned}
& \frac{1}{2} \frac{d}{d t}\|u\|_{H^{m}}^{2}+\mu\|\nabla u\|_{H^{m}}^{2} \\
& \leq\left|\int_{\mathbf{R}^{3}}\left(J^{m}(u \cdot \nabla u)-u \cdot \nabla J^{m} u\right) J^{m} u d x\right| \\
& \quad+\left|\int_{\mathbf{R}^{3}} J^{m}\left(\theta e_{3}\right) J^{m} u d x\right| \\
& \leq C\|\nabla u\|_{L^{\infty}}\left\|J^{m} u\right\|_{L^{2}}^{2}+C\left\|J^{m} \theta\right\|_{L^{2}}\left\|J^{m} u\right\|_{L^{2}} \\
& \leq C\left(\|\nabla u\|_{L^{\infty}}\|u\|_{H^{m}}^{2}+\|\theta\|_{H^{m}}^{2}+\|u\|_{H^{m}}^{2}\right) .
\end{aligned}
$$

Besides, we employ similar methods to (63) to the second equation of (1), yielding

$$
\begin{aligned}
\frac{1}{2} \frac{d}{d t}\|\theta\|_{H^{m}}^{2} \leq & \left|\int_{\mathbf{R}^{3}}\left(J^{m}(u \cdot \nabla \theta)-u \cdot \nabla J^{m} \theta\right) J^{m} \theta d x\right| \\
\leq & C\|\nabla u\|_{L^{\infty}}\left\|J^{m} \theta\right\|_{L^{2}}^{2} \\
& +C\|\nabla \theta\|_{L^{\infty}}\left\|J^{m} \theta\right\|_{L^{2}}\left\|J^{m} u\right\|_{L^{2}} \\
\leq & C\left(\|\nabla u\|_{L^{\infty}}+\|\nabla \theta\|_{L^{\infty}}\right)\|\theta\|_{H^{m}}^{2} \\
& +C\|\nabla \theta\|_{L^{\infty}}\|u\|_{H^{m}}^{2} .
\end{aligned}
$$


Combining (63) and (64),

$$
\begin{aligned}
& \frac{d}{d t}\left(\|u\|_{H^{m}}^{2}+\|\theta\|_{H^{m}}^{2}\right)+2 \mu\|\nabla u\|_{H^{m}}^{2} \\
& \quad \leq C\left(\|\nabla u\|_{L^{\infty}}+\|\nabla \theta\|_{L^{\infty}}+C\right)\left(\|u\|_{H^{m}}^{2}+\|\theta\|_{H^{m}}^{2}\right),
\end{aligned}
$$

and taking Gronwall inequality into consideration give

$$
\begin{aligned}
& \sup _{0<t<T}\left(\|u(t)\|_{H^{m}}^{2}+\|\theta(t)\|_{H^{m}}^{2}\right)+2 \mu \int_{0}^{T}\|\nabla u\|_{H^{m}}^{2} d \tau \\
& \quad \leq C
\end{aligned}
$$

Thus, we complete the proof of Theorem 1.

\section{Conflicts of Interest}

The authors declare that there are no conflicts of interest regarding the publication of this paper.

\section{Acknowledgments}

This paper was funded by the Science and Technology Research Program of Hubei Provincial Department of Education no. Q20161508 "Information Hiding Model Based on Statistical Characteristics of Image Carrier.”

\section{References}

[1] A. Majda, Introduction to PDEs and Waves for the Atmosphere and Ocean, Courant Lecture Notes in Mathematics, American Mathematical Society, 2003.

[2] J. Pedlosky, Geophysical Fluid Dynamics, Springer, New York, NY, USA, 1987.

[3] M. Cannone, "Harmonic analysis tools for solving the incompressible Navier-Stokes equations," in Handbook of Mathematical Fluid Dynamics, vol. 3, pp. 161-244, North Holland Publishing, 2005.

[4] C. Cao and J. Wu, "Global regularity for the two-dimensional anisotropic Boussinesq equations with vertical dissipation," Archive for Rational Mechanics and Analysis, vol. 208, no. 3, pp. 985-1004, 2013.

[5] A. Cheskidov and R. Shvydkoy, "The regularity of weak solutions of the 3D Navier-Stokes equations in $B_{\infty, \infty}^{-1}$, Archive for Rational Mechanics and Analysis, vol. 195, pp. 159-169, 2010.

[6] B.-Q. Dong and Z. Zhang, "On the weak-strong uniqueness of Koch-Tataru's solution for the Navier-Stokes equations," Journal of Differential Equations, vol. 256, no. 7, pp. 2406-2422, 2014.

[7] R. Temam, Navier-Stokes Equations, North-Holland, Amsterdam, The Netherlands, 1977.

[8] J. Fan, G. Nakamura, and H. Wang, "Blow-up criteria of smooth solutions to the 3D Boussinesq system with zero viscosity in a bounded domain," Nonlinear Analysis: Theory, Methods \& Applications, vol. 75, no. 7, pp. 3436-3442, 2012.

[9] H. Qiu, Y. Du, and Z. Yao, "Blow-up criteria for 3D Boussinesq equations in the multiplier space," Communications in Nonlinear Science and Numerical Simulation, vol. 16, no. 4, pp. 18201824, 2011.
[10] Z. Ye, "Blow-up criterion of smooth solutions for the Boussinesq equations," Nonlinear Analysis: Theory, Methods \& Applications, vol. 110, pp. 97-103, 2014.

[11] N. Ishimura and H. Morimoto, "Remarks on the blow-up criterion for the 3-D Boussinesq equations," Mathematical Models and Methods in Applied Sciences, vol. 9, no. 9, pp. 13231332, 1999.

[12] J. Fan and Y. Zhou, "A note on regularity criterion for the 3D Boussinesq system with partial viscosity," Applied Mathematics Letters, vol. 22, no. 5, pp. 802-805, 2009.

[13] Y. Jia, X. Zhang, and B.-Q. Dong, "Remarks on the blow-up criterion for smooth solutions of the Boussinesq equations with zero diffusion," Communications on Pure and Applied Analysis, vol. 12, no. 2, pp. 923-937, 2013.

[14] W. Wang, "Blow-up criterion for the zero-diffusive Boussinesq equations via the velocity components," Electronic Journal of Differential Equations, vol. 62, pp. 1-9, 2015.

[15] B. Dong, J. Song, and W. Zhang, "Blow-up criterion via pressure of three-dimensional Boussinesq equations with partial viscosity," Scientia Sinica Mathematica, vol. 40, pp. 1225-1236, 2010 (Chinese).

[16] B.-Q. Dong, Y. Jia, and W. Zhang, "An improved regularity criterion of three-dimensional magnetohydrodynamic equations," Nonlinear Analysis: Real World Applications, vol. 13, no. 3, pp. 1159-1169, 2012.

[17] B. Dong, S. Gala, and Z. Chen, "On the regularity criteria of the 3D Navier-Stokes equations in critical spaces," Acta Mathematica Scientia Series B, vol. 31, no. 2, pp. 591-600, 2011.

[18] Q. Chen, C. Miao, and Z. Zhang, "On the regularity criterion of weak solution for the $3 \mathrm{D}$ viscous magneto-hydrodynamics equations," Communications in Mathematical Physics, vol. 284, no. 3, pp. 919-930, 2008.

[19] S. Gala and M. A. Ragusa, "Logarithmically improved regularity criterion for the Boussinesq equations in Besov spaces with negative indices," Applicable Analysis, vol. 95, no. 6, pp. 12711279, 2016.

[20] S. Gala, Z. Guo, and M. A. Ragusa, "A remark on the regularity criterion of Boussinesq equations with zero heat conductivity," Applied Mathematics Letters, vol. 27, pp. 70-73, 2014.

[21] Y. Jia and B.-Q. Dong, "Remarks on the logarithmical regularity criterion of the supercritical surface quasi-geostrophic equation in Morrey spaces," Applied Mathematics Letters, vol. 43, pp. 8084, 2015.

[22] Y. Jia and B.-Q. Dong, "Asymptotic convergence rate of supercritical surface quasi-geostrophic equation in Morrey space," Journal of Mathematical Analysis and Applications, vol. 425, no. 2, pp. 1204-1213, 2015.

[23] P. G. Lemarié-Rieusset, Recent Developments in the NavierStokes Problem, Chapman Hall/CRC, Boca Raton, Fla, USA, 2002.

[24] J.-Y. Chemin, Perfect Incompressible Fluids, Oxford University Press, New York, NY, USA, 1998.

[25] Y. Meyer, "Oscillating patterns in some nonlinear evolution equations," in Mathematical Foundation of Turbulent Viscous Flows, M. Cannone and T. Miyakawa, Eds., vol. 1871 of Lecture Notes in Mathematics, pp. 101-187, Springer, 2006.

[26] T. Kato and G. Ponce, "Commutator estimates and the Euler and Navier-Stokes equations," Communications on Pure and Applied Mathematics, vol. 41, no. 7, pp. 891-907, 1988. 


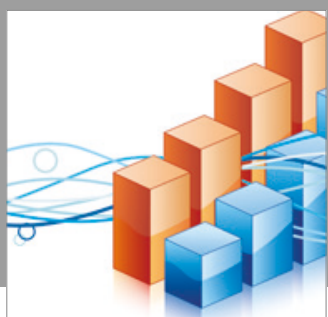

Advances in

Operations Research

vatersals

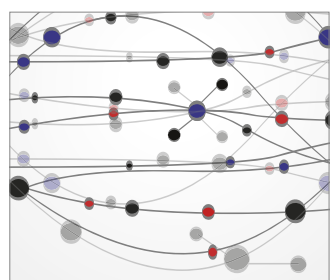

\section{The Scientific} World Journal
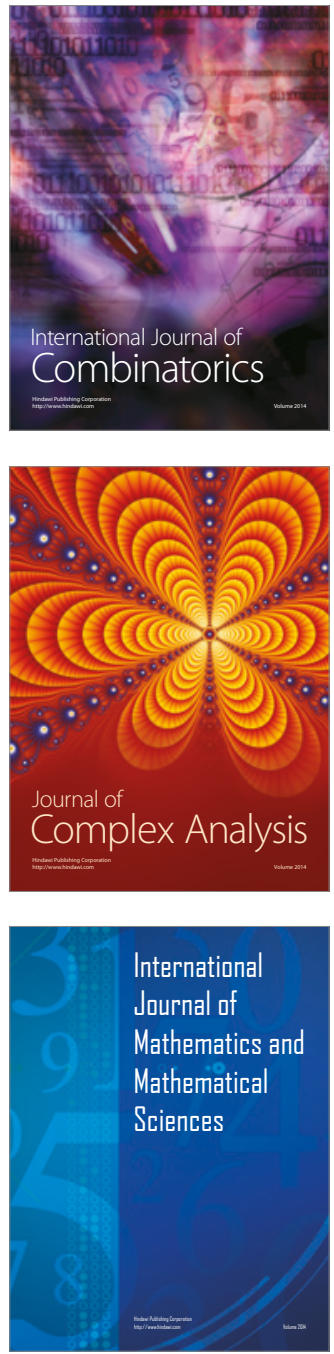
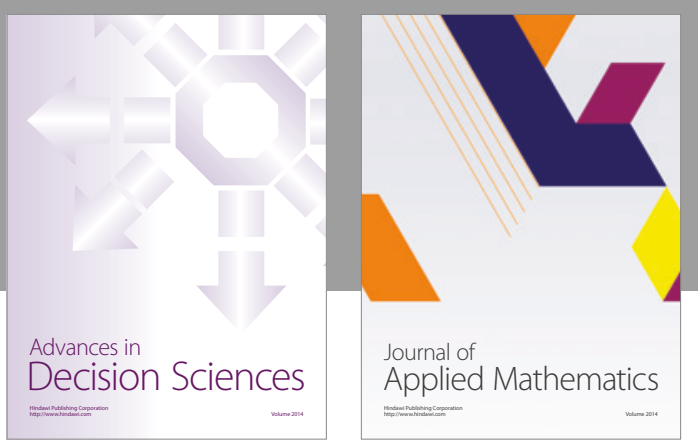

Algebra

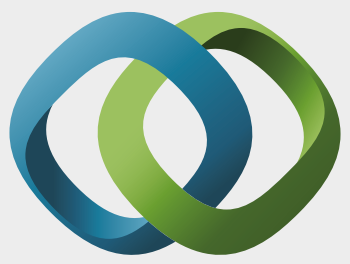

\section{Hindawi}

Submit your manuscripts at

https://www.hindawi.com
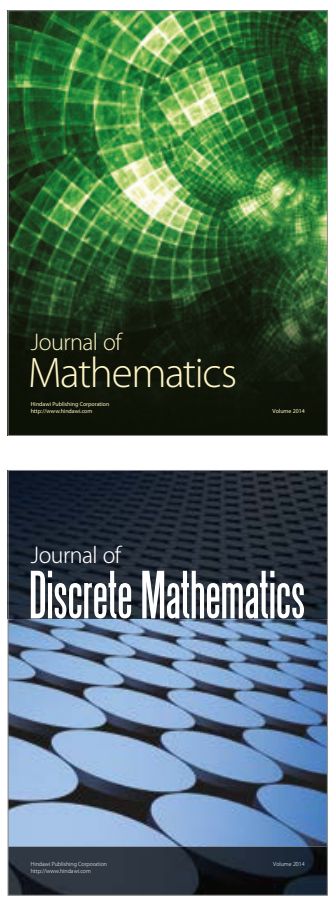

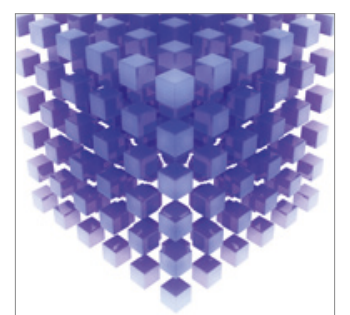

Mathematical Problems in Engineering
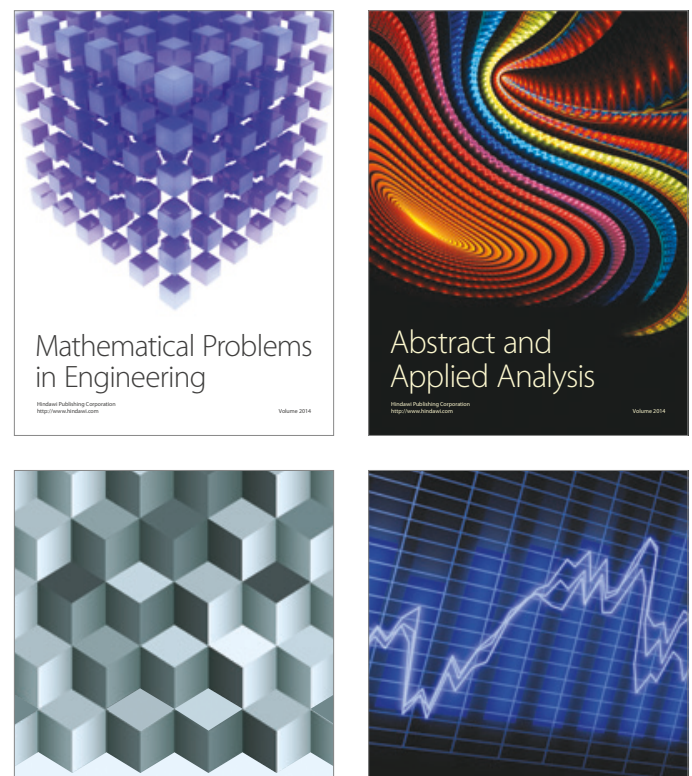

Journal of

Function Spaces

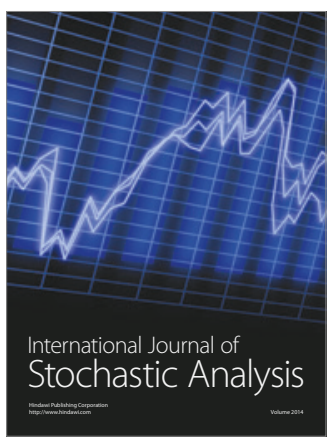

Probability and Statistics
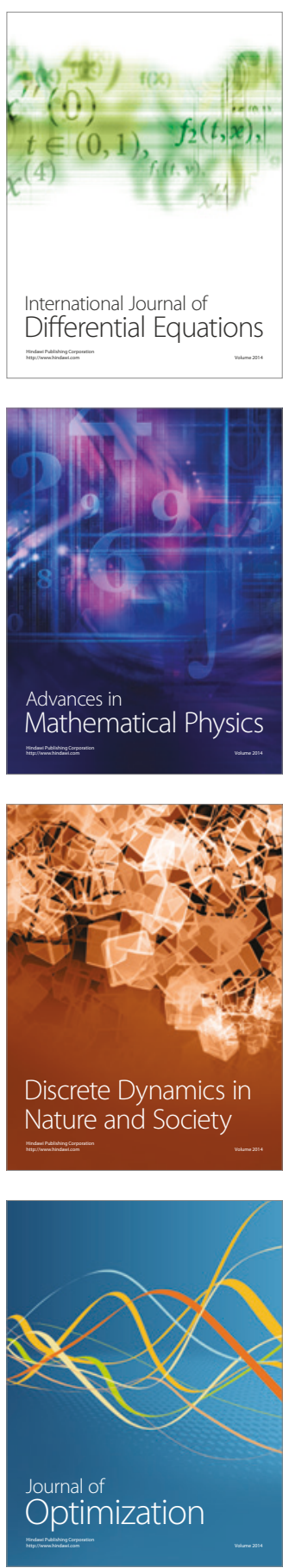